\title{
Política de formación de funcionarios públicos en Colombia: herramienta de modernización del servicio público
}

Nina Blanco Arias ${ }^{1}$

\section{Resumen}

La política de formación de los funcionarios públicos en Colombia, adoptada a través del Decreto 4665 de 2007 con el propósito de fortalecer la modernización de la gerencia pública en Colombia, ha sido ejecutada durante más de siete años. Su principal desafío fue implementar el modelo de: "capacitación por competencias", teniendo en cuenta las necesidades de las entidades territoriales en materia de formación, con el objetivo de mejorar la prestación de servicios en las entidades públicas del país. Para ello, el organismo competente realizó un ejercicio de diagnóstico de necesidades de formación en los territorios.

Basados en la política, se realizará un análisis con el objeto de conocer brevemente la estructura de la función pública en el país en aspectos como: los principales actores involucrados en su estructura y regulación, entre otros. Adicionalmente se planteará una reflexión sobre algunas circunstancias de la política, como la inexistencia de ejercicios de evaluación de resultado o impacto de sus objetivos, que permita identificar su contribución a la mejora del servicio público y la modernización del Estado, especialmente en los territorios, identificados como prioridad en el plan. administración pública, Candidata a Doctora en Ciencias Políticas y Relaciones Internacionales de la Universidad Complutense de Madrid, ex asesora y ex directiva en entidades públicas de control y supervisión en Colombia. Docente en Derecho Constitucional, Derecho Internacional y Responsabilidad Fiscal. Nina.blanco@hotmail.com 
El anterior análisis propone un cuestionamiento acerca de cuáles han sido los mecanismos y las herramientas que se han utilizado para formular, implementar y redireccionar la política durante su ejecución.

Esto permitirá la generación de conclusiones sobre su diseño, estructura, contextualización, características y la identificación de oportunidades de mejora, tomando como base los datos obtenidos de entidades de control fiscal territorial ${ }^{2}$. El análisis de esta información aproxima a la realidad de la gestión de la capacitación, y vislumbra escenarios relacionados con poblaciones que a pesar de hacer parte del servicio de la función pública, no son destinatarios de la política por circunstancias de orden legal y reglamentario.

\section{Introducción}

La formación de funcionarios públicos ha sido concebida como una herramienta para lograr la mejora de las administraciones públicas, propósito que se remonta a la noción de burocracia de Weber de inicios del siglo xx que denominó: "el saber profesional especializado" (Fleitas Ruiz, 2005). Esta noción partía de señalar que la administración pública requiere para su desempeño de un conocimiento profesional que le permita, al aspirante, a acceder por méritos al servicio público, y aplicar estos conocimientos en el desarrollo de sus actividades.

En Colombia, la política de formación de los funcionarios públicos fue adoptada a través del Decreto 4665 de 2007 con el propósito de fortalecer la modernización de la gerencia pública -política que ha sido ejecutada durante más de 7 años-y el principal desafío en su diseño fue implementar el modelo de "capacitación por competencias", haciendo énfasis en las necesidades de formación de las entidades territoriales. Para ello, el organismo competente realizó un diagnóstico de necesidades de capacitación y formación en los territorios, documentado en el informe de la primera fase del proyecto de 2006. Este estudio plasmó la situación de la capacitación de los empleados respecto al desarrollo de las competencias laborales y las necesidades regionales en esta materia (Departamento Administrativo de la Función Pública, 2006).

El Departamento Administrativo de la Función Pública (DAFP) yla Escuela Superior de Administración Pública (ESAP) son los órganos encargados de liderar esta política. El DAFP fue creado con la misión de liderar la modernización y el mejoramiento continuo de la administración pública en Colombia, así como promover el desarrollo

2 Información obtenida del Sistema de Información de la Auditoría General de la República, con autorización de uso para fines exclusivamente académicos. 
de los servidores públicos con el propósito de aumentar la legitimidad del Estado. En síntesis, es el organismo competente para fijar las directrices sobre modernización de la administración en sintonía con el mejoramiento del talento humano. Por su parte, la ESAP es el ente a cargo de la ejecución de la política de formación de los servidores públicos.

Las acciones del DAFP y la ESAP se remontan a 1998, año desde el cual han venido realizando un ejercicio de formulación de la política, obedeciendo a los objetivos trazados en los diferentes planes de desarrollo, a bien de lograr una reforma de la administración pública causada por los cambios introducidos por la Constitución Política de 1991.

Como antecedentes de la política se destaca el ejercicio realizado por el DAFP y la ESAP, en el que se llevó a cabo un diagnóstico de necesidades a través de la aplicación de una encuesta a 220 municipios del país. El análisis de los resultados arrojó la priorización de las principales temáticas de capacitación en el nivel municipal; acto seguido, se realizó una caracterización regional de necesidades y su validación, ejercicio que desencadenó en el documento actual de política pública, tal como se informa en sus antecedentes.

En este orden, la política estableció como objetivo la mejora de la calidad de la prestación de los servicios a cargo del Estado para el bienestar general y la consecución de sus fines; adicionalmente, fijó la necesidad de garantizar la instalación cierta y duradera de competencias en los funcionarios y las instituciones (Departamento Administrativo de la Función Pública, 2010). Sin embargo, atribuir de forma exclusiva a la formación de la tarea de lograr el mejoramiento y la calidad de los servicios a cargo del Estado resulta utópico si tenemos en cuenta otro tipo de factores que inciden en la prestación del servicio público y su calidad. Ese tipo de factores y circunstancias a que se hace referencia tienen relación con la asignación presupuestal, las situaciones de seguridad y el orden público en los territorios, entre otras causas.

\section{Modernización administrativa: mejora en la calidad del servicio público a través de la capacitación de los funcionarios}

La preocupación por la modernización administrativa incluye, dentro de sus fines, asegurar la calidad en el servicio público en busca de una mayor eficiencia y eficacia, generando una nueva cultura de la función pública que la acerque a las buenas prácticas del sector privado. Dentro de las acciones estratégicas se encuentra la profesionalización del talento humano en todos sus niveles (Ministerio para las Administraciones Públicas, 1992). 
A partir de la Constitución de 1991, la administración pública ha venido transformándose. Prueba de ello es la preocupación por mejorar la formación de sus funcionarios en búsqueda de la calidad, de acuerdo al objetivo principal de la Política.

En la formulación de los tres últimos Planes Nacionales de Desarrollo se pueden observar los esfuerzos emprendidos por los diferentes Gobiernos. Como se observará a continuación, Colombia ha evolucionado en la gestión del talento humano, sumando a la política de formación aspectos novedosos con el ánimo de adecuarla a la realidad del país.

El Plan Nacional de Desarrollo 2006 - 2010. Estado comunitario: desarrollo para todos (Departamento Nacional de Planeación, 2007) establece, en sus diferentes políticas, la necesidad de implementar acciones de modernización en sectores como: la fuerza pública, la inteligencia estatal, la salud pública, el sector social, los propósitos de seguridad alimentaria, la educación, el catastro y el registro, el transporte, los medios de comunicación, el sector financiero, la organización electoral, el servicio de justicia y el sistema penitenciario; todo esto en el marco de la institucionalización de la política de modernización del Estado.

En esta búsqueda define como destinatarios de la política de formación a los funcionarios del desaparecido Departamento Administrativo de Seguridad (DAS), a los funcionarios judiciales, a los docentes, a las autoridades ambientales y a los gestores jurídicos públicos. Estos últimos como consecuencia de la preocupación del Gobierno por el alto índice de demandas y pérdidas litigiosas a cargo del presupuesto estatal.

Por lo demás, apunta a la profesionalización del empleo público trazándose la necesidad de reforzar el proceso de reclutamiento, capacitación y estímulos del servicio civil. Así mismo, establece dentro de sus objetivos el diseño y la implementación del Sistema Nacional de Competencias Laborales que incluye, en su estructura, el diseño, la formulación y la implementación de fases de acreditación, así como la capacitación y la evaluación por competencias. Este esfuerzo se materializó más adelante con la expedición del Decreto 4665 del año 2007 que fijó la Política de Formación.

Por su parte, el Plan Nacional de Desarrollo 2010 - 2014 Prosperidad para todos (Departamento Nacional de Planeación, 2010) centra sus fines en la modernización en sectores como: seguridad y defensa nacional, infraestructura y transporte, tecnologías de la información y las comunicaciones, política de empleo y salud pública. Además, comparte los propósitos del plan previsto para el cuatrienio anterior pero suma importantes sectores como los organismos de control. Este último como consecuencia de la preocupación por combatir la corrupción. Adicional a sus esfuerzos, hace énfasis en la necesidad de fortalecer el sistema de atención y prevención de desastres, la política de empleo público y el sector administrativo de la función pública. 
En cuanto a la política de formación de los funcionarios públicos, llama la atención una iniciativa referida a la formulación e implementación de un plan de formación en gestión del riesgo, dentro del sistema de atención y prevención de desastres. Pero sobre todo, lo más inquietante es que se trata de una política a coordinar con el Ministerio de Educación Nacional, sin mencionar la participación de los órganos competentes en la formación de funcionarios; es decir, el DAFP y la ESAP. Llama la atención porque si bien es el Ministerio de Educación Nacional el ente rector de la educación colombiana, la formación de funcionarios en el marco de la modernización estatal está prevista en un sistema y una política específica a cargo de los órganos especializados y creados para tal fin. Esto conlleva a enfatizar en la necesidad de ejecutar la política de formación y capacitación de forma coordinada y así combatir la duplicidad de acciones y pérdida de recursos, generada por el traslapo de funciones en los diferentes órganos del Estado.

En esta oportunidad el plan realiza un avance importante centrando sus objetivos frente a la formación de los funcionarios públicos, en el principio de vocación por el servicio público en el marco del Buen Gobierno, destacando la necesidad de contar con servidores públicos con una sólida cultura ética y de servicio a la sociedad, así como en el desarrollo de competencias laborales para el eficaz desempeño de sus funciones y actividades en el puesto de trabajo, iniciativa que evidencia un esfuerzo del Gobierno por dirigir la gestión del conocimiento de los funcionarios hacia la superación de la corrupción, acercando la política a las realidades sociales.

En este propósito se establece la realización de una planeación estratégica del talento humano y la implementación de acciones como: revisar el sistema de evaluación del desempeño, implementar procedimientos que permitan comprobar las competencias de quienes ingresan al servicio público, fortalecer los sistemas de información del empleo público, modificar el esquema de control interno a fin de procurar la eficiencia de la administración, desarrollar conductas éticas como parte fundamental del Plan Nacional de Formación y Capacitación y potencializar el talento humano hacia la cultura del servicio en el marco de un Sistema Nacional de Gestión por Competencias Laborales en la búsqueda de la calidad de los servicios públicos, reiterando la finalidad última prevista en la Política de formación, esto es: la calidad en el servicio público.

Entre tanto, es oportuno detenerse en este propósito para llamar la atención sobre la importancia de generar metodologías que identifiquen el impacto de los resultados de la política en la calidad del servicio público. Hasta ahora, en las instituciones públicas, el ejercicio para la identificación del impacto de la capacitación ha dado un avance hasta la verificación del uso de los conocimientos adquiridos en el puesto de trabajo. Es así como el DAFP ha creado instrumentos como la Guía Temática para el desarrollo del Plan Institucional de Capacitación y la Guía para su Evaluación 
(Departamento Administrativo de la Función Pública, 2004), con el ánimo de orientar a las instituciones; sin embargo, no existe un instrumento conocido que permita evaluar el impacto de la política pública en la mejora de la calidad del servicio público y el desempeño de las instituciones, a pesar de ser una preocupación prevista en el documento de la política, que señala como obligación a cargo del DAFP y la ESAP: "La obligación de evaluar el impacto de la política de formación y capacitación, buscando precisar si su implementación ha permitido mejorar la prestación de los servicios a cargo del Estado, y si los empleados públicos han mejorado sus niveles de competencia" (Departamento Administrativo de la Función Pública, 2010, p. 47). Para esta obligación prevé la creación de un sistema de información que permita identificar los cambios ocurridos desde la implementación de la política, a través del registro de datos que relacionen los servicios prestados de manera deficiente o con oportunidades de mejora, los niveles de competencia de los empleados y los resultados de la evaluación de desempeño, entre otros.

Actualmente el Departamento Administrativo de la Función Pública opera el Sistema de Información y Gestión del Empleo Público (sigeP), el cual contiene información sobre el talento humano, convirtiéndose en una herramienta para las instituciones que les permite adelantar procesos como el Plan Institucional de Capacitación, de manera que su existencia evidencia las acciones del Gobierno para el recaudo de información que a futuro permita realizar una evaluación del impacto de la capacitación en la mejora de la instituciones públicas; no obstante, no se conoce un ejercicio que dé cuenta de una evaluación de este tipo a la fecha. Sin embargo, el Gobierno cuenta con las herramientas para el recaudo de su información; por lo tanto, es un reto la implementación de una evaluación de impacto para hacer uso eficiente de estos datos.

Es conveniente acentuar un llamado al Departamento Nacional de Planeación para ejercer, en el ámbito de sus competencias, la evaluación a las políticas públicas. El ideal es que estas evaluaciones se realicen por un órgano externo y no por el propio ejecutor de la política. Como es natural, esta circunstancia pone en riesgo la objetividad de la evaluación, sumado a que se trata de una tarea asignada a este organismo.

Por su parte, el Plan Nacional de Desarrollo 2014 - 2018 Todos por un nuevo país: Paz, Equidad y Educación (Departamento Nacional de Planeación, 2014) continúa marcando la modernización del Estado en el propósito del buen gobierno. Su dinámica realza la necesidad de fortalecer sectores como la educación, a la que le ha dado un énfasis especial en este cuatrienio, y de forma subsiguiente, las Tecnologías de información y comunicaciones (TIC), la infraestructura y transporte, la adecuación de tierras, la seguridad y defensa nacional, la policía judicial y la política fiscal, entre otros. 
En cuanto a la modernización del Estado en el marco del buen gobierno, establece como prioridad el fortalecimiento de capacidades del Estado en frentes estratégicos, para lo cual fijó la política nacional de eficiencia administrativa al servicio del ciudadano.

Por su parte, la modernización en la gestión del talento humano, enmarcada en los objetivos del buen gobierno, plantea como estrategia la consolidación del empleo público, para lo cual fija acciones como: el fortalecimiento de las competencias laborales, el desarrollo de estrategias de capacitación para el cumplimiento de los objetivos de las instituciones, la promoción del bilingüismo y la adopción de un modelo de competencias moderno que se complemente con un sistema de evaluación vinculado al desempeño institucional, punto en el que trata un tema importante, hace extensivas estas acciones a los servidores públicos vinculados mediante nombramiento provisional y empleos de carácter temporal. Esta problemática actual de la política será tratada más adelante ya que se trata de poblaciones que, por mandato legal, se encuentran excluidas de la política de formación.

Al centrarse en la capacitación, el plan le atribuye a la Escuela Superior de Administración Pública (ESAP) la tarea de revisar y actualizar la pertinencia de los contenidos y sus destinatarios, priorizando el uso de enfoques prácticos que se acerquen a la realidad e insiste en la reducción de brechas en los territorios. Adicionalmente, establece la exigencia de brindar apoyo integral a los funcionarios víctimas del conflicto armado y la generación de espacios de empleo público para la población reinsertada y desmovilizada, sumado a la cimentación de proyectos de capacitación en el marco de procesos de construcción de paz sostenible y hace énfasis en la generación de un proyecto de "formador de formadores" al interior del sector electoral y de registro.

En el actual Plan Nacional de Desarrollo se hace evidente un objetivo hacia el cual se deben conducir todas las políticas públicas. Se trata de la paz. Con esta claridad, vale la pena detenerse en dos aspectos reconocidos como necesidades específicas de la política. El primero es la generación de contenidos pertinentes en las capacitaciones, el principal instrumento utilizado para la detección de necesidades de formación de los funcionarios públicos es la realización de encuestas a los funcionarios y sus jefes, quienes expresan desde su punto de vista y conocimiento los requerimientos en materia de capacitación. Aunque la opinión de la población objetivo de la política es un insumo muy importante, se corre el riesgo de expresar una realidad parcial de las necesidades de capacitación debido a que el propio destinatario de este proceso pedagógico no está en la capacidad de conocer de forma integral todos los requerimientos formativos que le permitan brindar un mejor servicio público. Esta detección debe basarse en las especificidades del sector, el plan nacional del desarrollo, los planes estratégicos y de acción de las instituciones que operan en la 
misma línea de necesidades y demás circunstancias que determinan un verdadero y pertinente diagnóstico que, en conjunto, procuren la calidad del servicio público.

El otro aspecto tiene que ver con las evaluaciones de desempeño, convertidas en instrumentos que simplemente obedecen al cumplimiento de las obligaciones del jefe y los funcionarios, limitando su acción a un único propósito: dar cumplimiento a las normas que imponen su realización al alterar su verdadera función como instrumento de gestión del talento humano. Esta herramienta debería arrojar resultados e información determinante para una efectiva detección de necesidades de capacitación y evidenciar el impacto de la misma en el desarrollo y mejora del desempeño del funcionario.

\section{Población destinataria de la política. Exclusión legal de funcionarios vinculados en provisionalidad}

En el acápite anterior se observan las metas propuestas por los últimos tres Gobiernos, con posterioridad a la expedición de la Política de Formación de empleados públicos. No obstante, en la práctica no evidencian una mejora en la calidad de la administración porque no existe un instrumento que permita identificar este impacto y porque se presentan dificultades en la ejecución e implementación de la política.

A continuación se realizará un breve análisis de algunos datos obtenidos de organismos de control fiscal territorial, relacionados con la gestión de la capacitación en este mismo período de tiempo; es decir, 2007 a 2014. Estos datos son el resultado de la investigación realizada en el marco de una tesis doctoral cuyo propósito consiste en analizar y evaluar la política que para este ejercicio nos permitirán aproximarnos a la realidad de la gestión de la capacitación en los territorios, fundamentalmente en aspectos puntuales como el tratamiento dado a una parte de la población de funcionarios públicos que, a pesar de desempeñar una función pública, se encuentran excluidos por la ley como beneficiarios de esta política.

En este orden, el primer aspecto a destacar es la población beneficiaria de la política. Para ello es necesario detenerse en la organización y estructura de la función pública en Colombia, regulada por la Constitución Política en los artículos 122 a 133, Ley 443 de 1998, Ley 909 de 2004 y Ley 4a de 1992. A partir de esta regulación puede observarse que el concepto de función pública se refiere a la actividad estatal, desarrollada a través del desempeño de los servidores públicos en orden al cumplimiento de los fines estatales.

La meritocracia es regla general para definir el acceso al empleo público, lo que exige la gestión de concursos de méritos en los que se evalúan las competencias, los 
conocimientos y las aptitudes de los aspirantes, permitiendo con ello la selección de los mejores candidatos; sin embargo, como se observará más adelante, esta regla general permite algunas excepciones en la forma de vinculación a la función pública diferente a la carrera administrativa.

La actividad estatal se desarrolla a través del régimen laboral administrativo, el cual contempla diferentes categorías de servidores públicos según la forma de vinculación. Esta clasificación se observa en la figura 1.

Figura 1. Clasificación del empleo público

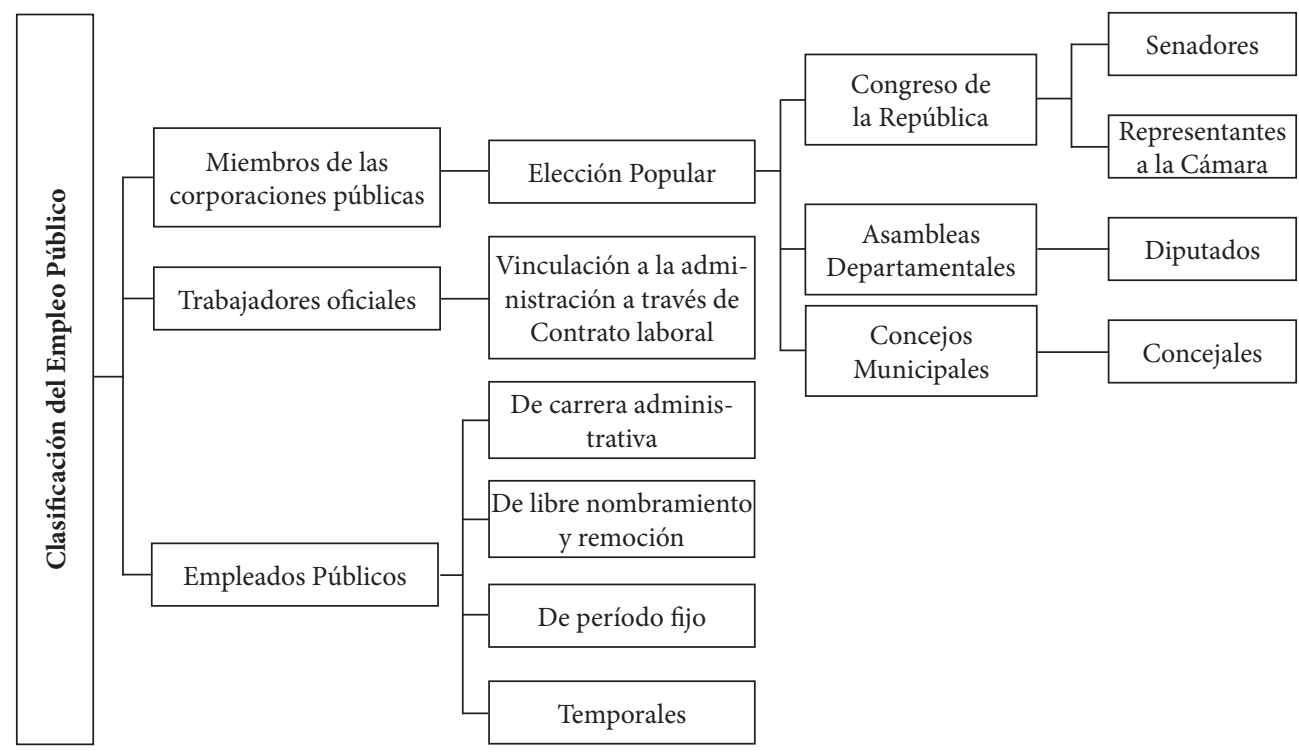

De acuerdo a lo observado, los servidores públicos se clasifican en: miembros de las corporaciones públicas, trabajadores oficiales y empleados públicos. Los miembros de las corporaciones públicas son los servidores de elección popular para conformar el Congreso de la República. Otros son los miembros de las asambleas departamentales y los consejos municipales.

Por su parte, los empleados oficiales comprenden dos clases: los empleados públicos y los trabajadores oficiales. La relación legal de estos servidores es de orden estatutario, tiene un período fijo y su retiro se produce una vez culmina el período para el cual han sido elegidos. Por otro lado se encuentra la categoría de trabajadores oficiales, cuya característica distintiva radica en que son vinculados 
a la administración pública a través de un contrato laboral, para prestar servicios generalmente en la construcción y mantenimiento de obras públicas, al igual que quienes prestan sus servicios para las empresas industriales y comerciales del Estado y las sociedades de economía mixta.

Es necesario detenerse en este aspecto y establecer la diferencia que existe entre los trabajadores oficiales y los contratistas del Estado a través de la figura de contrato de prestación de servicios. Los primerosse encuentran relacionados conla administración a través de un contrato de orden laboral, mientras que los segundos no tienen una relación laboral con el Estado, lo que conlleva, entre otras consecuencias, a la no existencia de subordinación, al igual que el no pago de prestaciones sociales. Estas son asumidas por el contratista como un trabajador independiente que se vincula de manera transitoria con la administración para prestar un servicio determinado.

Por último, la categoría de empleados públicos son aquellos que prestan sus servicios al Estado y están sometidos a un régimen legal y reglamentario; por lo tanto, su relación con la administración es de carácter estatutario. Dentro de los empleados públicos hay varias subcategorías determinadas por la forma de vinculación con la entidad, reguladas por el Artículo $1^{\circ}$ de la Ley 909 de 2004.

1. Empleados de carrera administrativa: se caracteriza principalmente porque el acceso y permanencia en el empleo se basa exclusivamente en el mérito, a través de la aplicación de procesos de selección que garantizan la transparencia e igualdad para sus aspirantes.

Existen ciertas entidades como las Fuerzas Militares y la Policía Nacional, la Fiscalía General de la Nación, la Rama Judicial, la Procuraduría General de la Nación, la Contraloría General de la República y las universidades estatales, en las que la regulación de su carrera administrativa se hace de forma especial por un reglamento que respeta los mínimos de la carrera general y se adecúa a las especificidades del empleo, generadas por la naturaleza y funciones de la entidad.

Adicionalmente, el Artículo $5^{\circ}$ de esta misma ley establece el empleo de "carrera administrativa" como la regla general con tres excepciones: los empleos de elección popular, los de libre nombramiento y remoción y los de dirección, conducción y orientaciones institucionales.

2. Empleados de libre nombramiento y remoción. Descritos por el al Artículo 5, numeral 2 de la Ley 909 de 2004.

3. Empleados de período fijo. Estos han sido tratados por la jurisprudencia en Sentencia T-137 de 2014.

“(...) Se hallan delimitados temporalmente por el término en que se haya concebido la labor, de manera que el funcionario puede ser retirado del cargo al momento en que se cumpla el plazo establecido para estos efectos. Entre los funcionarios 
que ejercen estos cargos se puede identificar al Contralor General de la República, Procurador Nacional, Defensor del Pueblo, Registrador Nacional, Magistrados, contralores departamentales y municipales, personeros municipales, entre otros. La jurisprudencia constitucional ha sostenido que uno de los propósitos del carácter delimitado en el tiempo se debe porque: "El período fijo, se explica más en virtud del principio democrático y de los principios de eficacia y eficiencia, que en gracia de asegurar al respectivo funcionario, un puesto de trabajo al margen de los vaivenes políticos (...) (Corte Constitucional, 2014) (Subraya y negrilla fuera de texto).

4. Empleados temporales. La Corte Constitucional los definió en Sentencia C-288/14:

“(...) Se trata de personas que forman parte de las listas de elegibles (Art. $3^{\circ}$, decreto 1227 de 2005) esto es, que superaron el concurso de méritos y esperan ser nombrados en el periodo de prueba que les permite acceder a la carrera administrativa; de ahí que su designación en un cargo de esta categoría significa la oportunidad preferencial de acceder a un empleo público en forma transitoria mientras se les nombra permanentemente en la planta de personal (...) (Corte Constitucional 2014).

La esencia del empleo temporal está en su transitoriedad, de lo cual se derivan otras diversas consecuencias, tales como: (i) no crea una vinculación definitiva con el Estado; (ii) no genera derechos de carrera administrativa; y (iii) está circunscrito exclusivamente a las labores para las cuales fue creado'”'

Esta categoría de empleo está regulada por el Decreto 1227 de 2005. Por último, a pesar de la posibilidad legal (Artículo 25 de la Ley 909 de 2004) de proveer los empleos de carrera administrativa a través del nombramiento de funcionarios en provisionalidad, ello no significa que se trate de una categoría más en el empleo público. Se trata de una forma de proveer empleos por vacancia temporal, en los casos en los que los titulares de los cargos de carrera administrativa se encuentren separados temporalmente de sus funciones por estar incursos en una situación administrativa.

La Corte lo ha explicado en los siguientes términos:

(...) Esta figura es una herramienta que permite a la Administración Pública suplir necesidades ante situaciones que generan insuficiencias de personal por vacancias temporales o definitivas, hasta tanto se provean estos cargos con los requisitos de ley o culmine la circunstancia que produjo la vacancia. No obstante, 
es necesario aclarar que la provisionalidad en el cargo no puede considerarse como un factor modificatorio de las calidades del mismo, toda vez que la estipulación legal prevalece sobre el escenario fáctico. De esta forma, la persona que asume este tipo de cargos, no es ajena a requerimientos técnicos y condiciones de calidad que se ordenan para ejercer una función determinada dentro del sector público. Los funcionarios que ejerzan funciones en esta modalidad, son beneficiarios de una estabilidad laboral intermedia o relativa que les permite ser valorados bajo criterios técnicos y no discrecionales, por ello no pueden equipararse a los cargos de libre nombramiento y remoción (...).

Sin embargo, como se mencionó, es necesario detenerse en esta población y situación de la función pública ya que en la realidad este tipo de vinculación se aparta de los postulados legales que pregonan una temporalidad. Se trata de una alto porcentaje de población, en algunos casos con más de 10 años de servicios a la administración, vinculados a través de "provisionalidad", desnaturalizándose en la practica la finalidad inicial de esta figura.

La dificultad existente respecto de la población de funcionarios vinculados en provisionalidad radica en que el Decreto 1567 de 1998 los ha excluido de las acciones de capacitación, amparando tal medida en el principio de prelación de los empleados de carrera administrativa. La norma señala que los empleados vinculados mediante nombramiento provisional, dada la temporalidad de su vinculación, solo se beneficiarán de los programas de inducción y de la modalidad de entrenamiento en el puesto de trabajo. Esta medida, adoptada en 1998 y ratificada mediante la Circular Externa 100-004 del 2010 del Departamento Administrativo de la Función Pública, desconoce la realidad actual del empleo público; en la práctica, estos empleados no ostentan la figura de provisionales, existen funcionarios vinculados en provisionalidad con más de ocho años de servicio en las entidades, años de servicio en los cuales han venido desempeñando sus funciones sin acceso a acciones de formación que les permitan mejorar su desempeño a través del fortalecimiento de las competencias laborales y, en consecuencia, incidir en el desempeño de sus instituciones. En la muestra obtenida de los funcionarios capacitados de las contralorías territoriales hasta 2014, previa la incorporación en carrera de funcionarios, gestionada en 2014 por la Comisión Nacional de Servicio Civil, se observa un alto porcentaje de funcionarios vinculados en provisionalidad, lo que se representa en la figura 2. 
Figura 2. Vinculación laboral de los funcionarios beneficiados con el fortalecimiento de competencias

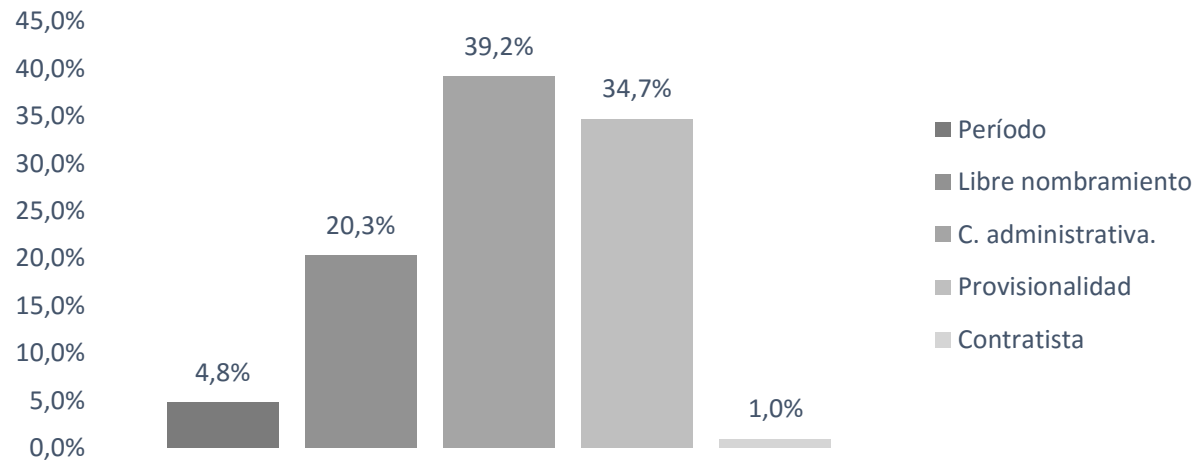

A esta problemática se suma la población de servidores vinculados a través de contratos de prestación de servicios profesionales de apoyo a la gestión. En la práctica se observan casos de personas cuyos contratos son renovados anualmente. Estas personas, en calidad de "contratistas", desempeñan una función pública pero no son objeto de acciones de formación y capacitación a pesar de ejercer dicha función pública. El cuestionamiento que surge de esta realidad es: ¿cómo incide la gestión de estas personas en la calidad de la prestación del servicio público?

Los datos analizados permiten afirmar que más de la tercera parte de los funcionarios que laboran en estas entidades han venido desempeñando sus funciones excluidos de la Política de Formación.

Lo anterior permite plantear una reflexión que, aunque ha sido mencionada en alguno de los Planes Nacionales de Desarrollo, aun no presenta una solución visible: ¿Es posible procurar calidad en la administración pública si un alto porcentaje de los funcionarios se encuentra excluido de las acciones de capacitación? ¿Qué tratamiento se da a esta población? ¿De cuántos funcionarios vinculados en provisionalidad estamos hablando? ¿Hasta qué punto es correcto hablar de provisionalidad?

Es claro que se trata de un fenómeno que requiere de la implementación de acciones que brinden una solución ya que indiscutiblemente todo aquel que preste un servicio público, aunque sea de carácter temporal, debe contar con la idoneidad exigida para el efectivo desempeño de sus funciones. Lo contrario sería afirmar que temporalmente está permitido prestar los servicios a cargo del Estado por parte de funcionarios que no cuenten con las competencias necesarias para su prestación. 


\section{Observatorio de capacitación y formación de lo público}

Otro aspecto importante en la ejecución de la política y que ha sido expresado en los Planes Nacionales de Desarrollo es la pertinencia en los contenidos y la oferta de formación hacia los funcionarios, que incide en la efectividad de la política y en la eficiente gestión de los recursos invertidos. Se trata de una exigencia que no se reduce a la satisfacción de las demandas de los funcionarios; esta obedece a los retos establecidos en la totalidad de políticas públicas plasmadas por el Gobierno en el Plan de Desarrollo, que recoge las circunstancias sociales del país.

Entre tanto, el Plan Nacional de Formación establece una guía temática que orienta a las entidades en la construcción de los planes institucionales de capacitación y define temas a priorizar; sin embargo, a pesar de ser un esfuerzo en la orientación de las instituciones está basada en el Informe de la Primera Fase del Proyecto 2006. Esto es casi una década hacia atrás. Este es precisamente uno de los retos de la pertinencia en los contenidos y deben obedecer a los principales objetivos planteados en los planes de desarrollo, al igual que a las políticas de Estado; por ejemplo, en la actualidad, el país se encuentra inmerso en un proceso ineludible hacia la consolidación de la paz, circunstancia que debe permear la política de formación, tal como se ha expresado en el Plan Nacional de Desarrollo actual.

Otra circunstancia que se observa es que se hace necesario enfatizar en la diversidad de sectores y especialidades que implica el servicio público. La temática actual prevista en la guía puede enmarcarse en los que algunos autores ha denominado formación general (Beltrán, 2002). Pero ¿qué pasa con la necesidad de consolidar una oferta de formación específica y adecuada a las singularidades de las instituciones y retos del país? Lo primero es concienciar a la administración de que prestan un servicio público todos aquellos que intervienen en esta prestación; por ejemplo, podemos traer a colación a quienes se desempeñan en la ejecución de políticas fiscales, de infraestructura y transporte, tecnologías de la información y las comunicaciones, y salud pública, por mencionar algunos sectores.

Es importante resaltar la definición de formación en asuntos públicos del Instituto Francés de Investigación y Debate sobre la Gobernanza (IRG), expuesto en las memorias del encuentro organizado por este Instituto y la Escuela Superior de Administración Pública (ESAP), el 15 de noviembre de 2008. Es importante esta definición puesto que no circunscribe el término "asunto público" a la simple administración pública, sino que extiende su entender a las actividades desarrolladas en la coproducción de los bienes públicos, por lo tanto, incluye a todos los actores de la sociedad civil que intervienen en este proceso (Instituto Francés de Investigación, Escuela Superior de Administración Pública ESAP, 2008). 
En la ejecución de la política en Colombia, esta especialidad en la oferta de formación busca gestionarse a través de los llamados Proyectos de Aprendizaje en Equipo (PAE), que promueven el aprendizaje colaborativo en pequeños grupos de funcionarios. Su objetivo es promover el intercambio de información y conocimiento a partir de su experiencia y aplicados a su realidad.

En la búsqueda de esta pertinencia las instituciones hacen un esfuerzo definiendolas competencias laborales en competencias comunes, funcionales y comportamentales. La figura 3 se explica cada una de ellas.

Figura 3. Competencias laborales generales que deben cumplir los servidores públicos

\begin{tabular}{|c|c|c|c|c|c|}
\hline 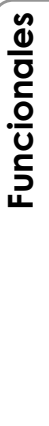 & $\begin{array}{r}\text { Precisan y detallan aquello } \\
\text { que debe estar en } \\
\text { capacidad de hacer el } \\
\text { empleado para ejercer un } \\
\text { cargo y se definirán a partir } \\
\text { del contenido funcional del } \\
\text { empleo. De esta hacen } \\
\text { parte los procesos de } \\
\text { formación y capacitación } \\
\text { en distintas áreas } \\
\text { temáticas: inducción, foros, } \\
\text { congresos, seminarios, } \\
\text { diplomados, } \\
\text { especializaciones, etc. }\end{array}$ & 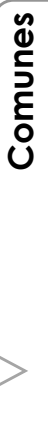 & $\begin{array}{r}\text { Hacen referencia al } \\
\text { conjunto de características } \\
\text { de la conducta que se } \\
\text { exigen como estándares } \\
\text { básicos para el desempeño } \\
\text { del empleo, atendiendo a } \\
\text { la motivación, las } \\
\text { aptitudes, las actitudes, las } \\
\text { habilidades y los rasgos de } \\
\text { personalidad. Se tienen: } \\
\text { orientación al resultado, } \\
\text { orientación al usuario al al } \\
\text { ciudadano, Transparencia, } \\
\text { Compromiso con la } \\
\text { entidad. }\end{array}$ & 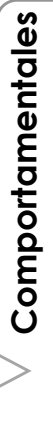 & $\begin{array}{r}\text { Se refieren a las actitudes, } \\
\text { los valores, los intereses y } \\
\text { las motivaciones con que } \\
\text { los funcionarios cumplen } \\
\text { sus funciones. Comprende } \\
\text { las siguientes:Ética, } \\
\text { liderazgo, trabajo en } \\
\text { equipo, compromiso social, } \\
\text { orientación al logro, } \\
\text { adaptación al cambio, } \\
\text { toma de decisiones, etc. }\end{array}$ \\
\hline
\end{tabular}

Sin embargo, se observa en la gestión de la capacitación de las entidades que la oferta de formación está destinada a formar funcionarios que trabajen en torno a protección y justificación legal de las decisiones y las responsabilidades de los funcionarios, de manera que se trata de una oferta de formación de base jurídica en su mayoría. Esta circunstancia ha tomado fuerza como consecuencia de los controles a los que están sometidos los funcionarios, de modo que uno de sus principales objetivos en el desarrollo de sus actividades es adecuar su actuar a la norma perdiendo de vista, en muchos casos, la finalidad última de la función.

En la población estudiada de los organismos de control fiscal territorial puede observarse el enfoque temático de las competencias funcionales como lo muestra la figura 4. 
Figura 4. Enfoques temáticos de las competencias funcionales 2007-2014 - contralorías territoriales

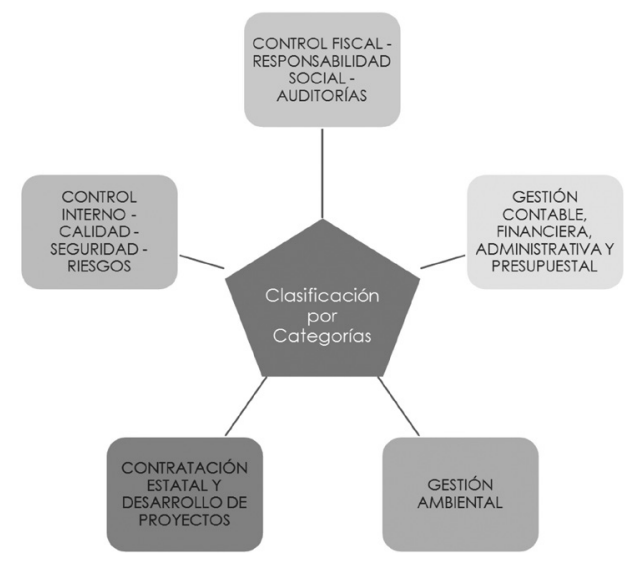

Fuente: elaboración propia

Esta reflexión permite referirse a la iniciativa emprendida por organizaciones como el Instituto de Altos Estudios en Asuntos Públicos (IDHEAP, Suiza), el Instituto de Estudios Políticos de París (Sciences Po, Francia) y el Centro para la Innovación y la Gobernanza Internacional (CIGI, Canadá), sobre la creación de un observatorio internacional de formaciones en asuntos públicos, surgida como consecuencia de la preocupación por mejorar la formación y capacitación de los funcionarios.

Lo expuesto nos lleva a resaltar la importancia de crear un instrumento que adopte esta buena práctica internacional. Se trata de la creación de un observatorio de formación de lo público, gestionado y operado por los organismos competentes, que entre sus funcionalidades permita realizar un ejercicio de monitoreo de la realidad del país y los retos hacia las cuales debe orientarse la formación de los funcionarios públicos. Esta iniciativa cuenta con una experiencia reciente en el país que puede revisarse en la gestión de un organismo de control fiscal ${ }^{3}$.

Se trata de un instrumento que permita capturar información y procesarla sobre aspectos como: funcionarios capacitados y en qué han sido capacitados, quiénes los han capacitado y las temáticas pertinentes para la satisfacciones de los problemas públicos entre otros temas relevantes. Con este enfoque se evitan dificultades como la duplicidad de esfuerzos y la pérdida de recursos públicos, entre otras circunstancias 
que permitirían una ejecución eficaz y eficiente de la política, además de la creación de metodologías que evidencien el impacto y los cambios producidos en la mejora de la calidad de las instituciones públicas.

Por otra parte, el logro de la pertinencia interactúa con el instrumento al vincularse con el logro de la calidad en la oferta de formación de funcionarios públicos. Para lograrla es preciso tener en cuenta varios aspectos a satisfacer: en primer lugar se debe asegurar la calidad de los docentes, quienes están formando a nuestros funcionarios. La motivación surge con el siguiente interrogante: ¿están ellos en primera medida capacitados para asumir una tarea tan importante? Es la misma discusión que gira en torno a la calidad y pertinencia en la política pública de educación. Esta pregunta nos lleva a otro cuestionamiento: ¿Cuáles son las instituciones que ejecutan la política? La Escuela Superior de Administración Pública (ESAP), tiene atribuida esta importante tarea; sin embargo, en la práctica, las instituciones públicas buscan colaboraciones de otras instituciones de educación, tanto públicas como privadas. Esto genera un riesgo respecto de la homogeneidad de la formación en lo público y los estándares existentes para garantizar su pertinencia y calidad.

La pertinencia en la formación y capacitación de lo público también implica estar alineados con los estándares mundiales y preparados para los retos de la globalización, esto implica el fortalecimiento de competencias respecto del manejo de una segunda lengua y el uso de las tecnologías de la información y las telecomunicaciones. Un ejemplo de estas exigencias encuentra sustento en el propósito del Gobierno de lograr el ingreso de Colombia a la Organización para la Cooperación y el Desarrollo Económicos (OCDE).

\section{Reflexiones sobre el análisis del diseño de la política y su necesaria evaluación}

El diseño de la política pública de formación de funcionarios en Colombia no presenta objetivos concretos (Pérez, 2015) respecto a las acciones previstas sobre su población y su efecto en la mejora de la prestación de los servicios de las instituciones, haciendo imposible verificar su contribución a los fines de modernización del Estado.

Los destinatarios de la política; es decir, los funcionarios públicos vinculados a través de carrera administrativa y libre nombramiento y remoción, presentan una gran diversidad en su formación, así como en características sociales y culturales, sector en el que se desempeñan y nivel regional en el que ejercen sus funciones, circunstancias que generan dificultades a la hora de la aplicación de la política y medición de la eficacia de sus resultados. 
La política pública de formación se implementa en las entidades a través del desarrollo de planes institucionales de formación (PIC) en cada vigencia fiscal, instrumento establecido por el DAFP y la ESAP a través de una metodología que debe ser operada por las oficinas encargadas de gestionar los recursos humanos; sin embargo, no existe una medición de sus resultados e impactos en la mejora del servicio de las instituciones. Lo anterior se genera por la falta de instrumentos de evaluación, el desconocimiento respecto de la incidencia de la adecuada gestión de la formación y su impacto en la gestión y el logro de sus objetivos, sumado la falta de aspectos motivacionales relacionados con la participación de los funcionarios en los programas de formación.

Adicionalmente, a pesar de que en la formulación de la política el Gobierno hace una gran esfuerzo por institucionalizar la formación como herramienta de modernización del Estado, su implementación es muy informal en relación con el cumplimiento de los aspectos fundamentales de la política como promover la formación por competencias con calidad por parte de sus prestadores. Esto ocurre porque la oferta en formación es muy variada en relación con sus prestadores; además de la ESAP, existen instituciones de educación de todo tipo, desde las más reconocidas hasta instituciones recién creadas que no cuentan con la infraestructura ni los recursos necesarios para prestar un servicio con calidad. Adicionalmente no existe un mecanismo de control que permita identificar estos vacíos en la aplicación de la política.

La anterior problemática es presentada por deficiencias en el diseño de la política, que se suman a problemas en su implementación. Las causas se refieren a la falta de definición de las acciones y su relación con el impacto en la mejora del servicio. Así mismo, no queda claro, en la formulación, la relación existente entre la implementación de los programas de formación y la mejora de las instituciones ni la forma en que realizará la verificación de su eficacia.

A pesar de que la política plantea la necesidad de desarrollar acciones con un enfoque diferenciado, establece una guía temática de carácter general para ser aplicada en todo el territorio, generando el desarrollo de programas de formación con las mismas características y dirigidos a poblaciones marcadas por una diversidad sectorial, regional, cultural, étnica y social. Lo anterior genera una ineficiente inversión de recursos, así como falta de pertinencia en los programas. Adicionalmente el planteamiento del enfoque temático se realizó en 2007, año desde el cual la política no ha sido actualizada a los nuevos objetivos y restos del país.

Otro de los aspectos problemáticos es que la política no presenta la inclusión de elementos motivadores para fomentar una verdadera participación de los funcionarios en los programas de formación y tampoco se asocia con mejoras salariales, crecimiento o desarrollo profesional y ascensos, entre otros. Tampoco 
presenta elementos de coerción que aseguren la participación de los funcionarios; la participación se verifica a partir de firmas en las listas de asistencia al programa de capacitación; sin embargo, este instrumento no permite comprobar si se realizó una efectiva instalación de competencias en el funcionario.

Sumado a lo expuesto, en el desarrollo de los programas de capacitación en las entidades intervienen todo tipo de instituciones de educación superior, las cuales en muchos casos colaboran con la administración a través de contratos de prestación de servicios, sin mediar ningún tipo de selección objetiva, situación que impide controlar la calidad de la institución y los programas ofrecidos. Así mismo, no se existe un mecanismo de control que verifique la calidad y el cumplimiento de los lineamientos de la política en los programas desarrollados.

La implementación de la política en las instituciones se realiza a través del Plan Institucional de Capacitación (PIC), el cual exige la realización de un diagnóstico de necesidades de formación para el planteamiento pertinente del programa de capacitación que debe seguir cada año la institución; no obstante, la realización de este diagnóstico es parcial puesto que deja de lado aspectos estratégicos dirigidos a la mejora de la institución; en su mayoría se basa en encuestas que contienen la opinión de los beneficiaros respecto de lo que estos consideran sus necesidades de formación.

Adicionalmente, el diagnóstico de la política plantea una problemática en cuanto a la existencia de brechas de capacidad institucional entre las entidades del nivel nacional y territorial; por lo tanto, resulta indispensable la definición de acciones financieras que permitan la reducción de estas brechas de manera que las instituciones con bajos niveles de capacidad institucional cuenten con pocas posibilidades de aumentar sus niveles y reducir sus diferencias frente a las entidades del orden nacional.

Por último, la política establece lineamientos técnicos y la creación de instrumentos para su correcta implementación en las entidades públicas, empero, este cumplimiento no está acompañado de control, lo que impide la verificación de sus resultados. Incluso, no se efectúa un correcto seguimiento a la política; es decir, de los programas que se desarrollan anualmente en las instituciones, lo que genera falta de fuentes de información para realizar una verificación del cumplimiento de los objetivos de la política.

\section{Conclusiones}

En el análisis se evidencia el esfuerzo realizado por el Gobierno desde la expedición de la Política Nacional de Formación de los empleados públicos. En los últimos tres Gobiernos son claros los avances planteados en la formulación de la política, 
que ha pasado de un planteamiento aislado de la política a su introducción como herramienta de "buen gobierno", a la vez que se observa una mayor conciencia respecto de la importancia de mejorar en la formulación y ejecución de la política como presupuesto necesario para imprimir calidad en la gerencia pública.

También se hace evidente que, después de aproximadamente siete años de ejecución de la política, no existen ejercicios de evaluación del impacto de la misma, por lo que se hace necesario contar con información que permita medir los cambios ocurridos en la gerencia pública colombiana, con ocasión de la implementación de la política desde 2007. Es importante diferenciar la medición de los resultados o impactos de la capacitación en la institución que demuestre los cambios sociales ocasionados con esta intervención.

Por lo tanto, se insiste en que el proceso de las políticas públicas requiere de la evaluación para su correcto diseño, ejecución y formulación, paso que se constituye en un reto para la Política Nacional de Formación. Después de ocho años de ejecución resulta imprescindible evaluar su impacto en la mejora de la calidad de la gerencia pública. En este orden de ideas se recomienda ejecutar una evaluación por un organismo externo al ejecutor que le brinde al Gobierno nacional la información necesaria para continuar de manera efectiva en el perfeccionamiento de la misma.

Los datos expuestos demuestran algunas de las circunstancias con oportunidades de mejora en la política. Este es el caso de la población excluida y el logro de la pertinencia en los contenidos.

La problemática existente en el empleo público ha generado la exclusión de las acciones de capacitación de una parte importante de la población de funcionarios públicos, por lo que se requiere de la implementación de acciones referidas a la mejora integral de la política de empleo público. Más allá de la política de formación y capacitación, la solución a esta problemática requiere la implementación de acciones integrales en todo el sector de la función pública.

En cuanto a la pertinencia de los contenidos, se avanza por el camino correcto pero no se puede perder de vista que las acciones públicas deben avanzar y evolucionar en torno a los problemas sociales y buscar su satisfacción. Por ello, se insiste en la necesidad de adecuar la política a la coyuntura actual del país; lo contrario es empezar a edificar sobre bases que resultan obsoletas y poco adecuadas a esa coyuntura.

Lo señalado es con el propósito de insistir en la importancia de efectuar una evaluación de impacto a la política pública de formación y capacitación que sin duda le proporcionará al Gobierno la información necesaria para adecuar la política a las 
necesidades actuales y evidenciar las diferentes oportunidades de mejora, así como las buenas prácticas que deben enfatizar las instituciones públicas.

\section{Referencias bibliográficas}

Albaladejo, G. (2014). Teoría y Práctica de las Políticas Públicas: Valencia (España), Editorial Tirant Lo Blanch.

Bañón, R. (2003) La Evaluación de la Acción y de las Políticas: Madrid (España), Editorial Díaz de los Santos.

Beltrán, M. (2002) “La formación y los empleados públicos”, en Revista Española de Investigaciones Sociológicas, 51-68.

Capacitación de Empleados Públicos para el desarrollo de competencias, Segunda Edición, 2010. Bogotá: Colombia.

Departamento Administrativo de la Función Pública, (2010) Plan Nacional de Formación y Capacitación de Empleados Públicos para el desarrollo de competencias, Segunda Edición, p. 47, 2010. Bogotá: Colombia

Departamento Administrativo de la Función Pública (2004), Plan Institucional de Capacitación: Guía para su evaluación, Grupo de Publicaciones y Recursos Educativos ESAP, 2004. Bogotá: Colombia.

Departamento Administrativo de la Función Pública (2006), Informe Primera Fase del Proyecto 2006: Situación de la Capacitación de los Empleados y Necesidades Regionales de Capacitación, 2006. Bogotá: Colombia

Departamento Nacional de Planeación, (2007), Plan Nacional de Desarrollo 20062010 Estado Comunitario: desarrollo para todos, Tomo I, Imprenta Nacional, 2007. Bogotá: Colombia

Departamento Nacional de Planeación, (2011), Plan Nacional de Desarrollo 20102014 Prosperidad para todos, Tomo I y II, Imprenta Nacional, 2011. Bogotá: Colombia.

Departamento Nacional de Planeación, (2014) Bases del Plan Nacional de Desarrollo 2014 - 2018: Todos por un nuevo país Paz, Equidad y Educación, Versión para el Congreso, nd, Bogotá. 
Escuela Superior de Administración Pública, (2013) Guíapara la Formulación del Plan Institucional de Capacitación-PIC- con base en Proyectos de aprendizaje en equipo. Bogotá: Colombia.

Harguindéguy, J. (2013). Análisis de Políticas Públicas. Tecnos (grupo anaya S.A.). 2013, Madrid.

Instituto Francés de Investigación y Debate Sobre la Bobernanza (IRG) y Escuela Superior de Administración Pública (ESAP) (2008), Memorias: La formación y capacitación suramericana en asuntos públicos, 2008, Bogotá: Colombia.

Lindblom, C. (1991) El Proceso de Elaboración de Políticas Públicas. Ministerio para las Administraciones Públicas, 1991, Madrid.

Pérez López, C. (2015) Técnicas de Evaluación de Impacto: Madrid (España), Editorial Ibergaceta Publicaciones S.L.

Pérez Sánchez, M. (2005). Análisis de Políticas Públicas: Granada (España), Editorial Universidad de Granada.

Subirats, J. (2012). Análisis y Gestión de Políticas Públicas: Barcelona (España), Editorial Planeta. 


\section{Epílogo}

El interés por los asuntos de Gobierno ocupa la atención de diversos grupos y opiniones que apuntan a la búsqueda de los métodos que hacen posible mejorar la dirección de la sociedad y la conservación del Estado. Dirigir la vida comunitaria es un conjunto de complejidades que se inscriben en condiciones objetivas del poder, las cuales articulan la relación de la sociedad con el Estado. La dirección de lo comunitario es un asunto de relevancia para entender y explicar el sentido del Gobierno, más aún de la gobernabilidad. Esta no es un objetivo deseado, sino imperativo para organizar lo público y lo colectivo con base en los rendimientos crecientes del poder.

La gobernabilidad alude a las capacidades que se producen para que la sociedad, la economía, las políticas públicas y la vida ciudadana logren un desempeño más eficaz que permita que los diversos sectores de la vida pública tengan las condiciones que facilitan la expansión y el desarrollo de la economía de mercado. En el tiempo presente, la conducción de la sociedad desde la esfera del Estado es un tema de relevancia, considerando que los problemas y conflictos tienen que atenderse y procesarse en un esquema que permita el diseño de las soluciones, así como el ejercicio inteligente del poder.

Ello implica armonizar intereses, expectativas, motivaciones y compromisos en la ruta de la eficacia política, la cual deriva de la capacidad que se tiene para generar la gobernabilidad democrática. El ejercicio del poder es la clave para situar los ámbitos de la gobernanza, más aún en la sociedad democrática. Los ámbitos mencionados apuntan a que exista una integración por cuestiones políticas, económicas, públicas, sociales y estatales. Cada uno de ellos tiene no solo su propia identidad, sino su impulso; situación que permite graduar los tiempos y el tiempo para llevar a cabo la construcción y funcionalidad de la democracia. Por tanto, la gobernabilidad es un imperativo de primer orden que no se debe soslayar porque se nutre de la realidad objetiva dada por actores, estructuras, procesos y acciones orientadas a cumplir fines deliberados, así como por la interacción de los grupos y organizaciones de la sociedad civil. 
Es importante destacar que la gobernabilidad es una condición para que la sociedad y el Estado armonicen programas y acciones que posibiliten el tratamiento más efectivo de las demandas en competencia, así como el curso de los problemas que se definen desde la esfera de las decisiones públicas. La importancia de tener condiciones idóneas para la conducción de la sociedad es una tarea que exige sentido de la estabilidad, equilibrio dinámico y eficacia en los procesos institucionales.

Por su parte, la estabilidad se enlaza con la correlación de fuerzas que hay en la comunidad y que es importante proteger con políticas y acciones que encauzan problemas y expectativas. El equilibrio dinámico significa que en la sociedad civil no hay punto de reposo y que los reacomodos constantes del poder es algo a destacar para evitar que se descarrile. En este orden de ideas, los procesos institucionales aluden a las reglas y procedimientos que se aplican para que el curso de los asuntos colectivos, a través de la agenda de Gobierno, sean canalizados hacia las instancias que se encargan del tratamiento y solución de los problemas públicos.

Por ello la gobernabilidad se sitúa como un estado necesario que ha de permitir que la sociedad no se consuma en sus conflictos ni en sus contradicciones, para lo cual se utilizan herramientas vinculadas a la naturaleza de los problemas públicos, así como de las soluciones que se diseñan con el concurso de dirigentes y cuadros burocráticos, tomando en cuenta los órganos de representación de la sociedad y evitando que prevalezca la visión piramidal del poder.

La sociedad civil contemporánea es más activa, estructurada, corresponsable y vigilante, motivo por el cual el poder del Estado no se impone per se, sino con base en consensos que se aplican con el método de las políticas públicas. Hoy gobernar no es equivalente al ejercicio abusivo del poder ni a las posturas que omiten que en cada grupo social hay ventajas para que pueda ser tomado en cuenta discutiendo y adoptando las decisiones del interés colectivo. Una lección de la crisis del autoritarismo es que no se puede imponer el poder del Estado con métodos violentos, sino atendiendo a la pluralidad de los actores y grupos de la sociedad.

El esquema vertical del poder no tiene en las condiciones actuales oportunidad de fructificar desde la visión de la centralidad política. Por el contrario, ha ingresado a la ley de los rendimientos decrecientes desde el momento en que se asumen posiciones contrarias a la democracia, por cuanto que esta exige deliberar y adoptar las decisiones públicas por consenso. Por otra parte, el éxito del poder no consiste en su capacidad instrumental sino en que sea aceptado como un sistema de ventajas y costos compartidos, lo cual indica que hay un cuadro básico de derechos y obligaciones que limitan la acción del Estado cuando se pretende rebasar el ámbito de las libertades civiles y políticas.

La gobernabilidad tiene como premisa un compromiso con la democracia con la vigencia de las libertades subjetivas privadas y las libertades públicas. Estas son 
el referente que los Estados tienen para no invadir la esfera de las personas ni de las organizaciones de la sociedad porque implicaría una violación de los ámbitos de actuación y seguridad que el poder está obligado a garantizar. En este caso, la visión del Estado que corresponde a la gobernabilidad democrática es el Estado de Derecho, el cual es considerado como la etapa de mayor desarrollo institucional dado que está diseñado para que funcione como una institución que invoca la razón política y la razón jurídica, entendidas como palancas que modulan su desempeño sobre el eje de la certidumbre institucional.

La gobernabilidad en el Estado de Derecho tiene importancia estratégica dado que considera factores políticos, jurídicos y económicos. Los factores políticos están dados por la vigencia de la democracia que reconoce derechos universales, libertades civiles y públicas, la representación política, la alternancia en el poder, el control del poder con transparencia y rendición de cuentas, así como la deliberación y argumentación, que son el binomio para llevar a cabo la discusión de los asuntos colectivos, considerando la tolerancia, la civilidad y el respeto por los demás.

Los factores jurídicos son cruciales porque las personas en la sociedad moderna reconocen la existencia de individuos entendidos como sujetos legales que tienen, para ello, un marco de derechos y obligaciones para ejercer las libertades civiles y políticas, sobre la base de la igualdad, a fin de evitar privilegios gremiales o corporativos que dañan las relaciones personales e interpersonales. Los factores económicos son importantes porque reconocen las libertades civiles para producir, comerciar, intercambiar e ingresar de lleno a las dinámicas de la economía de mercado, a fin de participar en la competencia de los agentes económicos, interviniendo en las zonas de riesgo que implican la competencia, la innovación y la acumulación del capital a la hora de invertir, pero que demanda certeza por parte de la autoridad para crear las condiciones de seguridad en términos de los derechos de propiedad y la reducción de los costos de transacción.

La gobernabilidad, por tanto, es un medio para diseñar los sistemas de instituciones que hacen factible la conducción de la sociedad, destacando tanto los incentivos positivos como los incentivos negativos.

Lo anterior porque la tarea de gobernar implica la definición de las reglas del juego -las instituciones- a fin de armonizar las implicaciones vinculadas con la funcionalidad en la relación de ganadores y perdedores. Si gobernar es en buena medida la adopción de decisiones para asignar costos, distribuir beneficios y atenuar los impactos en la vida de la sociedad.

La gobernabilidad en el Estado de Derecho es un conjunto de procesos que se construyen, mejoran y reproducen para dar factibilidad a la complejidad de la vida asociada. Es, por lo mismo, una categoría de análisis y comprensión indispensable 
para relacionarla con la eficacia que las políticas públicas han de generar, como estrategias deliberadas y orientadas hacia el cumplimiento de fines teleológicos.

Las políticas públicas y la gobernabilidad son herramientas inseparables para la mejor comprensión de lo que sucede en la sociedad civil. La gobernabilidad democrática se vincula con el modo de gobernar y este se sustenta en la expresión axiológica de lo que son las constituciones políticas. Para fines de eficacia, lo importante es que el modo de gobernar contribuya a que la sociedad, la economía y la vida colectiva tengan las mejores condiciones para que sean reproducidas con el auxilio de las políticas. En efecto, las políticas públicas son proveedoras de gobernabilidad dado que responden a la lógica del modo de gobernar. Tanto su diseño como su implementación es un asunto de capacidades directivas y operativas que tienen a su cargo que su desarrollo e implementación se efectúe de acuerdo a los objetivos y metas colectivos. Toda política pública provoca costos y beneficios en la vida colectiva. Lo importante, en términos de gobernabilidad, es destacar cómo la autoridad distribuye los costos fiscales, políticos, sociales y económicos, de modo tal que no genere reacomodos bruscos que puedan romper la normalidad de la propia sociedad. Como estrategias de gobierno, las políticas públicas responden al fin de gobernar mejor a la sociedad, asegurando que sus condiciones de vida sea favorables para impulsar la economía, redistribuir beneficios y erradicar la desigualdad social que aumenta la brecha entre los que más tienen y los que carecen de lo indispensable para vivir.

En este sentido, las políticas regulatorias tienen como objetivo fijar reglas para todos, en términos de derechos, obligaciones, costos y beneficios. Las políticas distributivas se caracterizan por su grado de incidencia e impacto en los diversos sectores de la sociedad para atenuar la expansión de la desigualdad social, regional, grupal y comunitaria. Además, las políticas de desarrollo involucran objetivos cualitativos relacionados con el aumento del bienestar y la equidad sociales; mientras que las políticas de estabilización procuran evitar que el aumento de los precios, el encarecimiento del dinero y el control de la oferta de bienes y servicios no paralicen a la economía.

Las políticas de crecimiento se encaminan a que la producción material de la riqueza sea mejor y superior al crecimiento de la tasa de la población. Las políticas públicas, en consecuencia, no son neutrales ni carecen de compromisos. Están comprometidas con el orden jurídico, político, económico y social de la democracia y, en consecuencia, su riqueza de valor orienta y determina las decisiones colectivas que adopta la autoridad política, como responsable y representante de la gobernabilidad democrática. Por ello, las políticas que no generan costos no existen y las que no provocan impacto tampoco; todas las políticas ocasionan no solo acciones, sino 
consecuencias que no conviene desconocer porque su fundamento con base en la gobernabilidad es contribuir a potenciar su eficacia y sentido de racionalidad.

La racionalidad de las políticas se nutre de los valores que proclama la democracia, así como de los valores que defiende el orden político y jurídico vigente. Los valores se entienden como la concepción que se tiene de la vida, lo cual permite configurar las estrategias de política pública en razón de intereses organizados, presiones ejercidas, recursos escasos y decisión política.

Por tanto, las políticas públicas tienen como punto de partida una concepción de valor que se ha de traducir en programas, objetivos, metas, recursos, personas y áreas de organización que, con reglas claras del juego -instituciones- lleven a cabo su cumplimiento. En este caso, la gobernabilidad democrática se nutre de las políticas públicas dado que la actuación y el desempeño de las autoridades desde un esquema de acción pública -actores gubernamentales, actores no gubernamentales- se conjuga para dar vida, sentido e implementación a las políticas públicas.

Ahora bien, lo que hace o deja de hacer el Gobierno incide en la calidad de la gobernabilidad democrática, lo cual obliga a destacar el contexto, los actores, los procesos, los tiempos, las restricciones y las acciones que se conjugan para articular la intervención de la autoridad pública en puntos vulnerables que están dañando el desempeño de la sociedad y la economía de mercado. El nexo gobernabilidad y políticas públicas es indisoluble. Es un nexo estructural que corresponde con el mundo de las decisiones, las acciones y la eficacia que se obtiene con el Gobierno en acción. Este no actúa solo, sino que se desenvuelve en ámbitos de presión, demandas, problemas y grupos de interés que no cesan en su movimiento y que constituyen un referente a considerar en la construcción y estabilidad de la gobernabilidad democrática.

La pluralidad y diversidad de la sociedad caracteriza a la vida democrática, circunstancia a destacar en el flujo de las políticas públicas dado que son el camino que permite al Gobierno intervenir en el espacio público, con sentido público. Lo público de la sociedad y lo público del Gobierno son esferas a puntualizar en la eficacia que demanda la gobernabilidad democrática, lo cual indica que los procesos institucionales no se ciñen al mundo del deber ser, sino al modo en que se desempeñan las autoridades de cara los desafíos de la vida colectiva.

Lo público de la sociedad y lo público del Gobierno se fortalecen en la medida en que las políticas públicas nutren a la gobernabilidad, ya que sin considerar esos elementos no es posible destacar cómo las propias políticas públicas son la herramienta a través de la cual se asignan recursos, se distribuyen costos, se amortiguan impactos y se articulan las formas de respuesta que se diseñan para desactivar los puntos críticos de la vida comunitaria. Lo público de la sociedad y lo público del Gobierno es la clave para dar origen y desarrollo a las políticas públicas, dado que estructuran 
oportunidades de elección, acciones deliberadas y fines orientados a lograr que la comunidad reactive su vida productiva y pueda superar los desajustes que alteran los equilibrios dinámicos que es necesario preservar. De este modo, las políticas públicas se conectan con la capacidad de gobernar y esta da vida a la complejidad activa de la gobernabilidad democrática.

El arte de gobernar no se agota en los patrones de la política, sino que toma en cuenta la importancia el arquetipo de las políticas públicas. En la sociedad civil hay acciones de gobierno que, en un sentido público, impactan el bolsillo de los ciudadanos, en las expectativas sociales y en el deseo de que las condiciones de vida sean mejores. El arte de gobernar tiene como una de las columnas vertebrales la eficacia de las políticas públicas que, por su grado de intervención, incidencia y aplicación en la sociedad, favorecen, modifican y transforman condiciones de vida. En el mundo contemporáneo es urgente que la visión, el contenido y la propuesta de las políticas públicas tiene que ser más efectivo. Temas como migración, pobreza, desigualdad, discriminación, inequidad, exclusión social y epidemias deben ser objeto de un abordaje más eficiente, lúcido y estratégico por parte de las autoridades constituidas que tienen a su cargo los sistemas de gestión pública.

El desarrollo de la globalidad exige mejores respuestas de gobierno y mayor efectividad de la gobernabilidad democrática, a fin de la regularidad de la vida en la sociedad se no solo continúa, sino que se caracterice por asegurar rendimientos crecientes desde la capacidad de gobernar.

En la actualidad, el futuro de las democracias es incierto porque hay desigualdades que laceran la vida comunitaria. En la medida que las crisis ahondan las disparidades de vida, disminuyen las oportunidades de un mundo mejor y más equitativo. Por su parte, el impacto de las crisis tiene que institucionalizarse con sentido de previsión y manejo estratégico de las mismas. Además, el gobierno de las democracias contemporáneas vive momentos de reto que deben abordarse con mejores capacidades institucionales y con mejores sistemas de gestión pública, a efecto de que las políticas públicas sean objeto de un mejor diseño y una eficaz implementación.

Esto implica que los Gobiernos tienen que ser más imaginativos y mejor capacitados para no fallarle a los ciudadanos y que estos no pierdan la motivación de vivir y convivir en las reglas de la democracia, que salvaguardan libertades, transparencia, rendición de cuentas, renovación democrática del poder, voto de castigo y voto de recompensa. La capacidad de los gobiernos es integral; es decir, tienen que considerar la totalidad de la sociedad civil para decidir el tipo de política pública que se han de adoptar para superar las restricciones públicas. Hay, en este caso, políticas regulatorias, promotoras y de fomento que aluden a modos en que se puede acelerar el bienestar de la sociedad civil. Por otra parte, hay políticas de crecimiento, desarrollo, estabilización y equitativas que destacan la variedad de herramientas 
a considerar en los esquemas del desarrollo de la sociedad y la economía. Todo ello exige claridad de fines y medios para que, en un ejercicio de gobernabilidad democrática, las diversas alternativas aplicadas puedan favorecer lo mejor de la vida individual, grupal y comunitaria.

El gobierno por políticas públicas es la base para situar y contextuar los alcances de la gobernabilidad democrática, en tiempos en los cuales la actividad ciudadana crece y proclama el lugar que le corresponde en los procesos institucionales. Desde una perspectiva de cambio y transformación institucional, el gobierno por políticas públicas debe tener inventiva para superar crisis, turbulencias e incertidumbres, así como los procesos de cambios que se generan con el inicio y final de los ciclos de vida.

La gobernabilidad democrática es condición para la mejor conservación del Estado y las políticas públicas abonan para el mejor desarrollo de la sociedad, en un esquema de incentivos, propuestas y riesgos compartidos. En este sentido, la capacidad de gobernar implica producir gobernabilidad democrática con el concurso de las políticas públicas. Por ello, la articulación de intereses se ha de encaminar para que la economía tenga un desempeño productivo que permita valorar las rutas a seguir en la búsqueda del bienestar individual y colectivo. Para ello es fundamental que la cooperación social se acentué como un sistema de compromisos que permiten generar objetivos y metas vinculados tanto a la gobernabilidad democrática como al carácter pragmático de las políticas públicas.

Entre tanto, la cooperación social y la correlación de fuerzas son un imperativo de primer orden para acreditar la vigencia de la capacidad para gobernar. En este caso, las políticas públicas tienen que valorarse como estrategias de buen gobierno que se orientan a transformar condiciones de vida y a impulsar la capacidad de gestión sobre la base de procesos de coordinación, implementación y evaluación.

\section{Ricardo Uvalle Berrones ${ }^{1}$}

1 Titulado con mención honorífica en la licenciatura de Ciencias Políticas y Administración Pública de la Facultad de Ciencias Políticas y Sociales (fCPYS) de la UNAM. Así mismo, realizó su maestría y doctorado en Administración Pública en la misma facultad y, por los correspondientes y destacados trabajos de investigación, recibió mención honorífica. 
Esta obra se editó en Ediciones USTA, Departamento

Editorial de la Universidad Santo Tomás. Se usó papel esmaltado de 300 gramos para la carátula y papel bond beige de 70 gramos para páginas internas.

Tipografía: Minion Pro. y Myriam Pro Impreso por Digiprint S.A.S.

2016 
El presente libro es un producto académico de expertos y profesores de trayectoria nacional e internacional que a partir de sus investigaciones han construido nuevas visiones sobre los retos y desafíos que implica las políticas públicas en la gobernabilidad, teniendo en cuenta los contextos estatales que requieren la generación de nuevas metodologías para su análisis.

Para ello es importante partir del concepto de que las políticas públicas son uno de los elementos principales que enmarca la gobernabilidad, y que además, permiten analizar una serie de retos $y$ desafíos que enfrenta el Estado para dar respuesta a las dinámicas coyunturales y estructurales propias de la administración pública.

Este libro presenta diferentes visiones sobre los factores que conciernen en el debate, análisis y reflexión en torno a los temas de participación ciudadana, la cooperación internacional, los DDHH, las migraciones, el papel de los funcionarios públicos, la relación existente entre gobernados y gobierno así como un modelo relacional de las políticas públicas. 